\title{
The transformation of values into prices on the basis of random systems revisited: reply to my commentators
}

\section{Bertram Schefold ${ }^{1}$}

Published online: 30 May 2020

(c) The Author(s) 2020

\begin{abstract}
This paper defends The Transformation of Values into Prices on the Basis of Random Systems, published in EIER, by answering to the Comments made in the same journal by Professors Mori, Morioka and Yamazaki. The clarifications mainly concern the justification of the randomness assumptions, the conditions needed to obtain the equality of total profit with total surplus value in the simplified one-industry system and the invariance of the results to changes in the units of measurement.
\end{abstract}

Keywords Marxian economics · Transformation problem - Capital theory - Sraffian economics $\cdot$ Profits and distribution

JEL Classification B14 $\cdot$ B24 $\cdot$ B16 $\cdot$ C65 $\cdot$ D57

\begin{abstract}
My paper in the journal Evolutionary and Institutional Economics Review, published by the Japan Association for Evolutionary Economics, on The transformation of values into prices on the basis of random systems revisited, had first being presented at a workshop on "Random System Solution of the Transformation Problem: Discussion with Prof. Bertram Schefold" organized by Professors Kiichiro Yagi and Yuji Aruka. The presentation was introduced by Kiichiro Yagi (2019), it was commented upon by Professors Yoshihiro Yamazaki, Kenji Mori and Masashi Morioka. The paper was published by the journal as Schefold (2019), and the comments appeared in the same issue as Morioka (2019), Mori (2019), and Yamazaki (2019), the comments having been revised in the light of the publication of my paper. I had briefly reacted to the earlier formulations of their comments at the workshop in footnotes in my paper as it was published. My reply will take up these earlier reactions. At the workshop, the commentators had read my earlier paper Schefold (2016a), which has remained the main basis for their reactions.
\end{abstract}

Bertram Schefold

schefold@wiwi.uni-frankfurt.de

1 Fachbereich Wirtschaftswissenschaften, RuW Fach 70, Johann Wolfgang Goethe-Universität, Theodor-W.-Adorno-Platz 4, 60323 Frankfurt, Germany 


\section{The questions}

I should like to thank Professors Kenji Mori, Masashi Morioka and Yoshihiro Yamazaki for their challenging comments and the editors of this journal for the opportunity to reply.

It remained undisputed that the Marxian interpretation of profits as redistributed surplus value is a central proposition in Marxian economic theory; the question was, whether a new explanation for the equality of aggregate profits and aggregate surplus value, based on random systems, is an adequate analytical reconstruction of Marx's ideas, whether it is a special case or whether it has a more general meaning, and how it relates to other interpretations. As a proposed solution to the transformation problem, obeying the condition profits equal surplus value $(P=M)$, it is clearly "more narrow than the conditions regarded as necessary by Marx or Engels and broader than modern critics, from Bortkiewicz to Sraffa, thought" (Schefold 2019 , p. 261). It is not a transformation in the literal sense, since labour values and prices of production are first calculated separately from the input-output data and then related to each other in such a way that the identities are confirmed, which were used by Marx in his attempt to derive the prices from values. My main concern in Schefold (2019) had been to discuss the context of this analytical reconstruction by looking at the principal Marxian propositions in which the condition $P=M$ plays a key role. Values are redundant for the calculation of prices, yet indispensable for the understanding of the meaning of this identity - if it holds. But the discussion of the commentators focussed more on the formal properties of the solution, and so my reply will have to go back to these.

All three criticise the special nature of my assumptions as if I had ever failed point out that special assumptions are needed to make $P=M$ a result that can be rigorously proved. Did I ever promise more? The claim was not to have found an absolute truth, but to have found a new solution of the transformation problem that sheds light on the Marxian interpretation of capitalist reality. And so I said, for instance:'It has turned out....that Marx's transformation is correct, after all, if the investigation is restricted (emphasis added-BS) to so-called random systems" (Schefold 2019, p. 267). The solution had been compared to others in Schefold (2016a). It is more general than the assumption that prices of production are proportional to labour values because the organic compositions of capital are equal. This condition is not needed. It is also shown that Sraffa once thought that the Marxian $P=M$ was correct as a rough statement about averages: the capitalists get a surplus which is essentially the same, whether it is looked at in terms of prices or in terms of values, and that Sraffa thought that such an idea could be made rigorous if the economy worked like a one commodity economy, like a corn-model, where it is clear which share of production goes to which class. But to render this idea rigorous, Sraffa thought that it was necessary to assume that the system was in standard proportions and that the capitalist received a share of the standard product. Here it was shown that this restriction could also be overcome - the system did not have to be in standard proportions and yet $P=M$ could be derived, but certain random properties had to be postulated. 
To understand why Marx could see profit as redistributed surplus value, it is necessary to show first why value may be visualized as a substance. The metaphor of 'substance' reminds the modern reader of the conservation laws for matter and energy in Newtonian physics over time. We do not find a substance in this strict sense in Marx, since the means of production must be revalued as values and prices change over time as the systems of production change with technical progress, and particularly and most drastically in periods of crises. The theory of the forms of value, which extends to the third volume of Das Kapital, shows only how the process of reproduction may be seen as a circulation of the value substance as long as no such revaluations take place. I have tried to show how this vision may be maintained, if prices of production are introduced, although, for the derivation of the price system taken in isolation, values are redundant. The view that profits are redistributed surplus value imposed itself for Marx, since he thought that prices originated from values. How can profits again appear as a substance related to surplus value, once both are separately derived in a value system on the one hand and in a price system on the other? It is the random character of the system, which allows this metaphorical view of a circulating value substance to reappear after the separate derivation of values and prices from the structure of use values had led to the insight that the same surplus, measured in terms of values and in terms of prices, could not generally be equal. On average, Marx thought, the quantity of the substance of value must be the same, looked at in terms of values and in terms of prices. There is no law of conservation of the substance if the system changes and employment changes. The vision can be maintained, however, if the structure of production persists and has random properties, for then $P=M$ holds. That was the point to be made, and it does not matter much whether the assumptions needed for 'randomness' are very special or a little special.

I believe that the future discussion should focus more on the interpretation of Marx and on the question, whether my analytical reconstruction can be regarded as an adequate expression of Marxian ideas, given our modern knowledge about prices of production. Kenji Mori's comment is divided into two parts, of which each takes up one aspect of this problem. Yamazaki and especially Morioka concentrate on the formal aspects of the underlying model, and this is also useful; how the model works must be clarified. They each have their objections and raise doubts. I think these can be dispelled by means of a reexamination of some central concepts. Whether I am successful or not in convincing my readers, I remain grateful to my commentators for having compelled me to reconsider the issues, which I shall now take up one by one.

I have argued (Schefold 2016b) that the introduction of randomness is important not only in relation to Marx but also to neoclassical theory, as it evolved in the last third of the nineteenth century. There is no transformation of values into prices in neoclassical theory, but there is an analogue to the proposition that profits are redistributed surplus value. The great change, which occurred in the transition from classical to neoclassical economics, consisted of an explanation of functional distribution between wages and profits in terms of supply and demand for factors of production. But to treat capital as a factor of production, demanded and supplied according to the level of the rate of interest, aggregate capital had to be given prior 
to the rate of interest so that effects of changes in the rate of interest on the value of capital had to be assumed away, and this is possible in conditions similar to those encountered when the rate of profit changes in Marx in such a way that the value of the surplus does not change. It thus appears that different economists in the nineteenth century shared the same simplified view about the relationship between distribution, prices and the valuation of capital. The view of the structure of production as random in large aggregates allows to represent this vision in both cases, Marxian and neoclassical. In both cases, the conditions hold only exceptionally, and that is a criticism of the theories concerned, but the analyses of those conditions allow better to understand the inner logic of the theories and to asses their potentials and their limitations in a pluralist approach to economics.

\section{Yamazaki: three important objections-three clarifications}

Yamazaki's comment divides into two parts. He discusses Marx's mathematics in the second and emphasizes the modern character of his approach to the interpretation of differentials in calculus. Insofar we are in agreement. My question was how this use of dialectics - for Engels, at least, affirmed the dialectical nature of Marx's approach to mathematics-related to the dialectical expressions Marx used in his treatment of the forms of value, which are relevant to the transformation problem. Yamazaki discusses only the mathematical side of this comparison, without looking at the character of the Marxian dialectical treatment of the forms of value, and we can leave it at that.

The first part of his comment is concerned with one-industry systems. They are the determinate counterparts to the random systems under consideration in my paper. It should be clear that the random systems are the real object of the analysis and that the one-industry systems are useful only as a drastic simplification-one might speak of a caricature, which is useful because it represents some properties of random systems, which result in the limit if they grow large. He says (Yamazaki 2019, p. 321): "The elements are changing randomly in random matrices... nondominant eigenvalues disappear when their average is taken." He reminds us of applications of random matrices in physics, which describe fluctuating or uncertain states. This is different from the application in economics, for which we use the Goldberg-Neumann theorem. In the economic application, large input-output systems show in each row the industrial use of the commodities produced in other sectors for the production of the commodity characteristic for the sector represented by the row. The main assumption is that the inputs are independently and identically distributed with a mean specific for the row, and the intuitive justification for the assumption is that the specificity of the technical determinants relating inputs and outputs become less relevant as one disaggregates more and more and looks at the same time at the system in the large. In making this assumption, one tries to capture a property of large systems: what seems determinate and special in the small appears as accidental in the large. The specific structure of individual industries thus is abstracted from, and one looks only at their relative importance, represented by 
the mean of the distribution. The assumption that the distributions are i.i.d. is not sufficient to guarantee that the non-dominant eigenvalues will disappear for large systems; additional assumptions about the variances of the distribution on each row and the covariances between the rows have to be made, but since these assumptions are only sufficient and not necessary, I preferred to replace these assumptions by the simpler and more direct assumption that the non-dominant eigenvalues disappear. An economist may perhaps be pardoned for this. The assumptions do not exclude a great deal of variation of the coefficients in the small and, in particular, "many" input coefficients may be equal to zero.

This contrasts with the one-industry system as the determinate analogue. Here one asks how a semi-positive matrix looks like, which, as such, will have a left-hand side and right-hand side Frobenius eigenvector, if it is postulated that all non-dominant eigenvalues disappear. One finds that, given the dimension $n$, the most general form of such an input-output matrix is given by a positive matrix $\mathbf{A}$ of rank 1 and order $n$. It follows that it can be written as $\mathbf{A}=\mathbf{c f}$, where $\mathbf{c}$ and $\mathbf{f}$ are a column and a row vector respectively, and where $\mathbf{c}>0$ and $\mathbf{f}>0$. The random matrix, by contrast, may contain zeroes and its determinant will in general not vanish. For the nondominant eigenvalues only approach zero for large $n$; they are in general not equal to zero for any finite $n$. That the distribution of the input coefficients is i.i.d. implies that $\mathbf{f}=\mathbf{e}$, where $\mathbf{e}=(1, \ldots, 1)$ is the vector which we use as summation vector also.

Yamazaki seems to believe that the restriction $\mathbf{f}=\mathbf{e}$ implies a loss of generality; he writes that such a matrix is "very trivial" (Yamazaki 2019, p. 322). The opposite is true. Matrix $\mathbf{A}=\mathbf{c e}$ summarizes important properties of random matrices. Whether there are random matrices such that the determinate counterpart can be written as $\mathbf{A}=\mathbf{c f}$ has not even been proved.

I formulated this as a hypothesis in Schefold 2013, where I introduced A $=\mathbf{c f}$ as a one-industry system (associated with a labour vector, of course), and the properties of the one-industry system have since been explored-with useful additions by Morioka, as we shall see. But why should all industries be similar in that the input structure is on average given by $\mathbf{f}$ and not by $\mathbf{e}$ ? It is a more intuitive assumption that, if we want to speak about random distributions of inputs in the context of input output matrices at all, that the distribution is identical. I became interested in $\mathbf{A}=\mathbf{c f}$, because of an extension, which however has not been really explored so far: $\mathbf{A}=\mathbf{c f}$ implies that all industries have the same pattern expressed by $\mathbf{f}$. So, perhaps, in a random system corresponding to this one-industry-system, all industries would be somewhat like the steel industry in the era of coal and steel, and all sectors would be characterized by services supported by information technologies in another era. But to affirm that all industries are similar to only one industry-apart from random aberrations - is exaggerated. If this kind of investigation were to be pursued seriously, one would have to try to approximate the sectors of the economy by superpositions of a smaller set of industrial structures, a set larger than one and smaller than $n$; that would lead to a new research program. In conclusion, I should like to defend the random matrices in which the distribution of coefficients is i.i.d. as the best first approximation. The assumption is an abstraction, as always in economics. It is meant to grasp a characteristic, which is realistic only to some degree. I have given examples of empirical input-output systems with non-dominant eigenvalues, 
which do cluster somewhat around zero but by far not as strictly as do random matrices generated by computers (Schefold 2013, p. 961).

The reader will have understood that random matrices, taken by themselves, will not lead to linear wage curves or to the property that profits can be explained as a redistribution of surplus value; assumptions about the labour vector and its connection with the matrix are needed. To this end, I introduce a comparison between the actual vector of activity levels and a standard vector, proportional to Sraffa's standard commodity on the one hand, and between the labour vector and a so-called Marx vector on the other. Yamazaki takes issue with the latter. He interprets it as the vector of prices obtained at the maximum rate of profit of the system and says: "The wage rate here is zero. This is in complete contradiction to Marx's opinion" (p. 320). But this is a misrepresentation of my definition. I defined the Marx vector as that labour vector which, if it were the labour vector pertaining to matrix $\mathbf{A}$, would lead to prices being equal to labour values. This Marx vector $\mathbf{x}^{\mathbf{1}}$ is subject to strong normalization. Let $\mathbf{x}^{\mathbf{1}}, \ldots, \mathbf{x}^{\mathbf{n}}$ be $n$ linearly independent right-hand side eigenvectors of $\mathbf{A}$.

The labour vector $\mathbf{I}$ then can be represented as a linear combination of these eigenvectors. Eigenvectors are determined only up to a linear scale factor. We choose these scale factors in such a way that $\mathbf{l}$ is the sum of the normalized eigenvectors, hence $\mathbf{l}=\mathbf{x}^{\mathbf{1}}+\ldots+\mathbf{x}^{\mathbf{n}} \cdot \mathbf{x}^{\mathbf{1}}$ here is the real-valued right-hand side Frobenius eigenvector of $\mathbf{A}$. Hence we may interpret the difference $\mathbf{l}-\mathbf{x}^{\mathbf{1}}$ as the deviation of the real labour vector from that labour vector, which would lead to the equality or proportionality of prices and values. We shall interpret this difference $\mathbf{v}=\mathbf{l}-\mathbf{x}^{\mathbf{1}}$ further when we discuss Morioka's contribution. Here let us only note that $\mathbf{v}$ is a measure of the deviation of prices from values, given matrix $\mathbf{A}$.

We now come to an essential point. As the reader will have noticed, the condition $\bar{v}=0$ plays an essential role in the proof that $P=M$ and, by the way, also in the proof that wage curves of random systems are linear, hence in the application of the present approach to the critique of capital theory. The magnitude $\bar{v}$ here is the average of the components of vector $\mathbf{v}$, and we shall have to discuss the meaning of "average" in this context below. If $\bar{v}=0$, it means that the labour effort in the actual system is on average equal to the labour effort in a system with the same input-output matrix, but with a labour vector such that prices are proportional to labour values. It, therefore, can be shown that $\bar{v}=0$ implies that prices are on average equal to labour values, given the numéraire. Hence $\bar{v}=0$ is an important proposition, and it now turns out that $\bar{v}=0$ is a result, which can be proved, if the coefficients of the random system are i.i.d. on each row, hence if the random system corresponds to a one-industry system of the form $\mathbf{A}=\mathbf{c e}$, while $\bar{v}=0$ must be introduced as an additional assumption if $\mathbf{A}=\mathbf{c f}$. Indeed, one can even show that $n \bar{v}=0$ (or tends to zero with large $n$ in random systems) in a one-industry system of even capital composition, and this seems so important that I here repeat the short proof:

We have by our definitions

$$
n \bar{v}=\mathbf{e v}=\mathbf{e}\left(\mathbf{l}-\mathbf{x}^{1}\right)=\mathbf{e}\left(\mathbf{x}^{2}+\cdots+\mathbf{x}^{\mathbf{n}}\right) .
$$

Now, if the system is of even capital composition, we have 


$$
\mathbf{e A}=\mathbf{e}(\mathbf{c e})=(\mathbf{e c}) \mathbf{e},
$$

hence $\mathbf{e}$ is the Frobenius left-hand eigenvector of $\mathbf{A}$ and is orthogonal on each of the $\mathbf{x}^{\mathbf{2}}, \cdots, \mathbf{x}^{\mathbf{n}}$, hence $n \mathbf{e v}$ must be zero. We may conclude that the average $\bar{v}$ tends strongly to zero in random systems for larger $n$, as we get the convergence of the non-dominant eigenvalues to zero. The result that the labour effort is on average the same for any given labour vector and for the special labour vector, for which values and prices are equal, given the random input-output structure, is thus characteristic for random systems, for which the input coefficients on the rows are i.i.d.

Professor Yamazaki concludes (p. 323) that my derivation depends on "special" matrices.

I should say that it depends on random matrices in their purest form. He speaks about a "riddle" using the vector $\mathbf{v}$, because it allegedly refers to the difference "between labour input vector and the standard prices". I hope to have clarified that it refers to the difference between the labour vector of the actual system and that labour vector which would lead to prices being equal to values. He says that "the economic interpretation is difficult". It is difficult only because he has changed the definition.

\section{Morioka: activity analysis or the method of classical economics?}

In the first part of his contribution, Morioka concentrates on a formal analysis of one-industry systems and offers some original extensions. His criticisms are somewhat similar to those of Yamazaki; I shall try to answer them in the same vein. He then turns to the measurement of the quantities involved in the second part. I think that his negative conclusions are mistaken and due to an error in methodology, but I agree that the question is important, that I did not discuss it in my more recent papers on random systems, and I am, therefore, grateful to the editors of the journal for giving me the opportunity to fill a lacuna on the occasion of writing this comment.

Morioka discusses the one-industry system in the form $\mathbf{A}=\mathbf{c f}$. As I have tried to explain above, the idea of the random system is best captured with random systems of even capital composition, the determinate counterparts of which are given by $\mathbf{A}=\mathbf{c e} \mathbf{e}=(1, \ldots, 1)$ because random systems have been introduced to represent the idea of an economic system which is so large that the individual characteristics of industries disappear and there is only a random fluctuation of inputs so that the determinate counterpart reduces to a very simple structure; the rows are not only proportional, but each input is equal to the mean of the row. We also know: vector c is the capital composition and vector $\mathbf{f}$ the distribution of capital. These vectors are at the same time the left-hand side and the right-hand side eigenvector of $\mathbf{A}$, with

$$
\mathbf{A c}=(\mathbf{c f}) \mathbf{c}=\mathbf{c}(\mathbf{f} \mathbf{c})=\mu \mathbf{c}, \quad \mathbf{f} \mathbf{A}=\mathbf{f}(\mathbf{c f})=(\mathbf{f} \mathbf{c}) \mathbf{f}, \mathbf{f} \mathbf{c}=\mu=\frac{1}{1+R},
$$


where $R$ is the maximum rate of profit. The labour vector $\mathbf{I}$ is again written as the linear combination of the right-hand side eigenvectors $\mathbf{l}=\mathbf{x}^{1}+\ldots+\mathbf{x}^{n}$ and the activity level vector $\mathbf{y}$ is represented by the linear combination of the left-hand side eigenvectors $\mathbf{y}=\mathbf{q}_{1}+\ldots+\mathbf{q}_{n}$, so that

$$
\mathbf{v}=\mathbf{l}-\mathbf{x}^{1}=\mathbf{x}^{2}+\ldots+\mathbf{x}^{n}, \quad \mathbf{m}=\mathbf{y}-\mathbf{q}_{1}=\mathbf{q}_{2}+\ldots+\mathbf{q}_{n},
$$

I now call $\mathbf{v}=\mathbf{I}-\mathbf{x}^{1}$ the labour balance vector and $\mathbf{m}$ the standard balance vector. We saw above that the labour balance vector is the difference between the actual labour vector of the system and that labour vector which would cause the system to have prices proportional to values. In the same way, $\mathbf{m}=\mathbf{y}-\mathbf{q}_{1}$ is the standard balance vector, that is, the difference between the actual activity level vector and an activity level vector which would cause the system to be in Sraffa's standard proportions. Because of the strong normalization of the eigenvectors such that the coefficients of the eigenvectors in the linear combination in which they represent $\mathbf{l}$ and $\mathbf{y}$ respectively are all equal to unity, $\mathbf{x}^{1}$ and $\mathbf{q}_{1}$ are normalized so that

$$
\mathbf{x}^{1}=\frac{1}{\mu} \mathbf{A l} \quad \mathbf{q}_{1}=\frac{1}{\mu} \mathbf{y} \mathbf{A}
$$

This follows from $\mathbf{A l}=\mathbf{A}\left(\mathbf{x}^{1}+\ldots+\mathbf{x}^{n}\right)=\mathbf{A} \mathbf{x}^{1}=\mu \mathbf{x}^{1}$, since the eigenvalues pertaining to $\mathbf{x}^{2}, \ldots, \mathbf{x}^{n}$ are equal to zero. The same holds for $\mathbf{q}_{1}$.

I had introduced $\mathbf{x}^{1}$ as the Marx vector and $\mathbf{q}_{1}$ as the Sraffa vector. We now can write the labour balance

$$
\mathbf{v}=\mathbf{l}-\mathbf{x}^{1}=\mathbf{l}-\frac{1}{\mu} \mathbf{A l}
$$

this means that the labour balance is for each component equal to the difference between the direct labour used in the sector concerned and the direct labour embodied in the means of production, the latter being reduced by $1 / \mu$. The labour balance, therefore, is similar to the organic composition of capital. The organic composition of capital shows the inverse of the relation of the expenditure on direct labour to the expenditure on other means of production, measured in labour values. Instead of a ratio, we here have a difference, and instead of measuring in terms of labour values, we measure in terms of direct labour. Similarly, we obtain the standard balance

$$
\mathbf{m}=\mathbf{y}-\mathbf{q}_{1}=\mathbf{y}-\frac{1}{\mu} \mathbf{y A}
$$

Note that one can also write

$$
\begin{aligned}
& \mathbf{x}^{1}=\frac{1}{\mu} \mathbf{A l}=\frac{1}{\mu} \mathbf{c f l}=\mathbf{c} \frac{\mathbf{f l}}{\mathbf{f} \mathbf{c}}, \\
& \mathbf{q}_{1}=\frac{1}{\mu} \mathbf{y A}=\frac{1}{\mu} \mathbf{y c f}=\mathbf{f} \frac{\mathbf{y c}}{\mathbf{f c}}
\end{aligned}
$$


This array of formulas, which may look bewildering to the newcomer, will, I hope, clarify the price relationships for readers of the paper and the comments. As a first application, lets reconsider standard prices. From

$$
\mathbf{p}=\rho(\mathbf{A p}+w \mathbf{l})=\rho \mathbf{A p}+\rho w \mathbf{l}
$$

we can infer that Marxian prices, with the wage advanced, are equal to Sraffa prices in terms of any numéraire, if we replace the wage $w$ ex ante by the wage ex post $\rho w$. In the paper, to obtain the linear wage curve, we had normalized $\mathbf{f} \tilde{\mathbf{p}}=1$; these prices $\tilde{\mathbf{p}}$ become Sraffa prices, which pertain to his standard if we normalize labour so that $\mathbf{f l}=R /(1+R){ }^{1}$

We get immediately for the standard prices

$$
\tilde{\mathbf{p}}=\rho \mathbf{c}+\rho \tilde{w} \mathbf{l}
$$

Therefore,

$$
\rho \tilde{w}=\frac{1}{\mathbf{f l}}(1-\rho \mathbf{f} \mathbf{c})
$$

With $\mathbf{f} \mathbf{c}=1 /(1+R)$ and the normalization of $\mathbf{I}$, this yields Sraffa's linear wage curve in the familiar form

$$
(1+r) \tilde{w}=1-\frac{r}{R}
$$

It gives for the Marxian prices in this standard

$$
\tilde{\mathbf{p}}=(1+r)\left(\mathbf{c}-\frac{1}{R} \mathbf{l}\right)+\frac{1+R}{R} \mathbf{l}=\mathbf{l}+\mathbf{c}+\frac{r}{R} \mathbf{v}
$$

That standard prices are a linear function of the rate of profit had been shown before in my earlier papers, and Morioka has added an interesting different derivation. The present new formulation shows that these prices are equal to values expressed as direct labour $\mathbf{I}$ and indirect labour $\mathbf{c}$, if the rate of profit is zero, and that they then rise or fall linearly according to whether the labour balance is positive or negative. And it is here also obvious that these prices are equal to values on average (one only has to multiply the equation from the left with e). Prices remain equal to values if and only if the labour balance vector is zero.

We now get to more controversial points. Instead of standard prices, we consider the Marxian prices with the normalization $\mathbf{y} \overline{\mathbf{p}}=1$, where $\mathbf{y}$ are the given activity levels; $\mathbf{y}$ is in general different from e. With this numéraire and the normalization or measure of labour again being given by $\mathbf{f l}=R /(1+R)$, prices $\overline{\mathbf{p}}$ can be shown to be equal to (the derivation is in principle the same as in Schefold 2019, p. 294)

$\overline{1}$ This normalization was erroneously written as $\mathbf{f l} \mathbf{l}=1$ in footnote 17 in Schefold (2019, p. 293). 


$$
\overline{\mathbf{p}}=\frac{(1+R) \mathbf{l}-(1+r) \mathbf{v}}{(1+R) \mathbf{y} \mathbf{l}-(1+r) \mathbf{y} \mathbf{v}}
$$

It is clear that each component of the price vector is, as a function of the rate of profit, a hyperbola.

The question now is how the value of the surplus vector $\mathbf{s}$ changes with the rate of profit:

$$
\mathbf{s} \overline{\mathbf{p}}(r)=\frac{(1+R) \mathbf{s} \mathbf{l}-(1+r) \mathbf{s} \mathbf{v}}{(1+R) \mathbf{y l}-(1+r) \mathbf{y} \mathbf{v}} .
$$

This result entails the following propositions:

(1) The value of the surplus vector also is a hyperbola as a function of the rate of profit, hence $\mathbf{s} \overline{\mathbf{p}}(r)$ is either monotonically rising or monotonically falling, unless the hyperbola degenerates to a constant. This means that $P=M$ if and only if $\mathbf{s} \overline{\mathbf{p}}(r)$ is constant (with $r>0$ ). As Morioka has observed it, I did not clarify this fact in earlier presentations. If, in the general case, the components of $\overline{\mathbf{p}}(r)$ and hence $\mathbf{s} \overline{\mathbf{p}}(r)$ are significantly more complicated than a hyperbola, $\mathbf{s} \overline{\mathbf{p}}(r)$ may cut the constant $\mathbf{s} \overline{\mathbf{p}}(0)$ at several positive rates of profit $r_{i}>0$.

(2) We here have been considering hypothetical variations of the rate of profit. The actual or equilibrium rate of profit is that at which the profits so obtained are just adequate to buy the surplus, for distribution is determined by the given necessary wage and the remaining surplus. We, therefore, have two conditions, the wage rate is such that labour employed can buy the necessary wage, $\bar{w} \mathbf{y l}=\mathbf{b} \overline{\mathbf{p}}$ or $\bar{r}=P /(K+W)$; the fulfilment of each condition guarantees the fulfilment of the other. It is conceivable in the general case (but not likely) that $\bar{r}=r_{i}$, where $r_{i}$ is one of the rates of profit at which the curve $\mathbf{s} \overline{\mathbf{p}}(r)$ cuts the constant $\mathbf{s} \overline{\mathbf{p}}(0)$. It follows that plausible solutions of the transformation problem presuppose that $\mathbf{s} \overline{\mathbf{p}}(r)$ is a constant. This, in turn, means that $P=M$ will be observed only if $\mathbf{A l}$ is not regular, e.g. if the main neoricardian theorem does not hold (Schefold 2019, p. 288).

3) Hence we must seek conditions that are not only sufficient, but, as Morioka insists, also necessary for $\mathbf{s} \overline{\mathbf{p}}(r)$ to be constant. Following Schefold (2019, p. 294) we get with the new normalization

$$
(1+r) \mathbf{s v}(1+R) \mathbf{y l}=(1+r) \mathbf{y v}(1+R) \mathbf{s} \mathbf{l}
$$

which is equivalent to

$$
\frac{\mathrm{sv}}{\mathrm{sl}}=\frac{\mathrm{yv}}{\mathrm{yl}}
$$

and this an equivalent to 


$$
(\mathbf{s v y}-\mathbf{y v s}) \mathbf{l}=0
$$

the latter equivalence holds because $\mathbf{I}>0$.

It is now clear that $P=M$ will hold if,

(a) the labour balance is zero or the labour theory of value works with $\mathbf{v}=0$.

(b) The surplus is proportional to the activity level vector. If we write this condition as $\mathbf{s}=\alpha \mathbf{y}$, and if $\mathbf{b}$ is the necessary wage, so that total output $\mathbf{y}$ divides into $\mathbf{y}=\mathbf{y A}+\mathbf{b}+\mathbf{s}$, the condition will be fulfilled if $\mathbf{y}(\mathbf{I}-\mathbf{A})-\mathbf{b}=\mathbf{s}=\alpha \mathbf{y}$

$$
\mathbf{y}=\frac{\mathbf{b}}{1-\alpha}\left(\mathbf{I}-\frac{1}{1-\alpha} \mathbf{A}\right)^{-1} .
$$

It is, therefore, possible but not necessary that the economy is in standard proportions in this case. It presupposes $\alpha<R /(1+R)$. Morioka (2019, p. 307) has noted that this condition is different from the condition for prices to be expressed as linear functions of the rate of profit.

Both sufficient conditions (a) and (b) also hold for general input matrices and are not specific for the discussion of random or one-industrial systems-in case (b) we get in general:s $\overline{\mathbf{p}}(r)=\alpha \mathbf{y} \overline{\mathbf{p}}(r)=\alpha$. The composition is not that of the standard commodity, as Sraffa had thought (Schefold 2019, p. 299).

We now turn to the consideration of sufficient conditions, which are meant to complement the assumptions of randomness. We had indicated economic reasons why

$$
\begin{gathered}
\operatorname{cor}(\mathbf{m}, \mathbf{v})=\operatorname{cov}(\mathbf{m}, \mathbf{v})=0 \\
\operatorname{cor}(\mathbf{s}, \mathbf{v})=\operatorname{cov}(\mathbf{s}, \mathbf{v})=0
\end{gathered}
$$

Morioka observes that

$$
\mathbf{y} \mathbf{v}=\left(\mathbf{q}_{1}+\mathbf{q}_{2}+\ldots+\mathbf{q}_{n}\right)\left(\mathbf{x}^{2}+\ldots+\mathbf{x}^{n}\right)=\left(\mathbf{q}_{2} \mathbf{x}^{2}+\ldots+\mathbf{q}_{n} \mathbf{x}^{n}\right)=\mathbf{m v}
$$

This allows modifying the transformation in Schefold (2019, p. 295):

$$
0=\operatorname{cov}(\mathbf{m}, \mathbf{v})=\mathbf{m v}-n \overline{m v}=\mathbf{y v}-n \overline{m v}
$$

From $\bar{v}=0$ (which is a result if $\mathbf{f}=\mathbf{e}$, as we saw above, and otherwise an assumption), we can conclude $\mathbf{y v}=0$ and, by an analogue reasoning, $\mathbf{s v}=0$; the vanishing of the correlations thus implies $P=M$.

Morioka indicates that these sufficient conditions are not necessary. The observation is correct but important only if economic reasons can be given why the necessary and sufficient conditions should hold. Since $\mathbf{s}, \mathbf{y}$ and $\mathbf{I}$ are positive vectors, we do not have to worry that the nominators in the proportion should be zero. The mathematical expression can be rendered in words as: The labour balance of the surplus should relate to the direct labour of the surplus as the labour balance of total production to the direct labour of total production. But what is the economic meaning 
of this condition, except that it implies the desired result? By contrast, the correlation or covariance condition has the independent meaning that, where we have large matrices, it is a reasonable abstract (i.e. not directly realistic) assumption to expect normal distributions that are independent. Similarly, $\mathbf{m}$ and $\mathbf{v}$ are of different origin, so that a correlation, if one was found, might be regarded as spurious and one would be challenged to explain it by a hidden variable. But even if these a priori estimates should turn out to be drastically wrong in empirical investigations, they retain some importance from the point of view of the history of economic thought as potential explanations of what Marx meant by his "tendency" for $P=M$ to hold and as an explanation of what was meant by "average". That he had a vision of simplified relationships for economic systems in the large is confirmed by the fact that such visions also were shared by his contemporaries like Jevons or Böhm-Bawerk who, for their neoclassical assumptions, also needed an economic structure that had to be simpler than the complications that Sraffa found in his analysis of capital theory.

Morioka's attitude seems to be to let that go, because he ultimately objects that the construction presented with the one-industry systems, be they of even composition or not, is worthless, because they are, according to him, not invariant to changes in the measurement units. This is an important point which has not been raised before in the discussion, and I have worried about such matters for a long time myself.

At first sight he seems to be completely right. What does average price or average value mean, if the average is taken across different commodities? We know what average price means in Marx: it is the average price of one commodity in one industry, where the producers use different methods of production so that their costs are different. Marx assumes in general that commodities are homogenous with a few exceptions such as the case when commodities are adulterated like bread when the baker is using flour of inferior quality. This indicates that commodities are homogenous in classical theory not simply because they are chemically homogenous, but because there are institutions and a social consent that define their quality, and one must insist on the word "social" because one needs to emphasize the contrast with a definition of homogeneity based on the judgement of utility-maximising individuals. Marx refers in the beginning of Das Kapital to the "commercial knowledge of commodities". It was a discipline which taught which properties a commodity had to have to be accepted in the trade. Given this long history of the idea of homogeneity, it seems absurd to speak of averages of prices across industries. We speak of averages of temperature across a number of days or of averages of weight across a number of persons, but what is the average of prices across different commodities? This is the question Morioka asks when he shows how averages and covariances seem to depend on the units of measurement.

If gross output, equal to the activity level vector, is described by a vector such as

$$
\left(150 \frac{\mathrm{kg} \text { corn }}{\text { year }}, 50 \frac{\mathrm{pigs}}{\text { year }}, 100 \frac{\mathrm{loil}}{\text { year }}\right)
$$

the average of the pure numbers, here 100 , would be economically meaningless. Morioka is right to postulate that "any sound economic analysis requires that the 
assumptions therein do not depend on the choice of the measurement units of each product". With this, he tries to reduce my analysis ad absurdum, but, in so doing, he violates the assumptions which are made by Marx according to classical economic theory. The analysis of the value system uses a given structure of quantities, expressed by the input-output matrix $\mathbf{A}$, the labour vector $\mathbf{l}$, the vector of gross outputs $\mathbf{y}$ and the necessary wage $\mathbf{b}$, so that the surplus $\mathbf{s}$ results. To be derived are values, prices and the equilibrium rate of profit $\bar{r}$, at which profits buy the surplus. The gold price is derived as well, so that values and prices can be expressed in terms of gold, as in Schefold (2019, p. 277). The comment by Kenji Mori encouraged me to go more deeply into the question of how to represent the production of gold. Morioka now stimulates me to look more closely at the measurement units.

We begin with the labour balance $\mathbf{v}=\mathbf{l}-\mathbf{x}^{1}$; the vector is composed of two labour vectors: the one corresponding to the actual labour in this system and $\mathbf{x}^{1}$, which is, as we saw, the hypothetical labour vector, with which prices would be equal to values and the organic composition of capital uniform. Hence the problem of dimension for $\mathbf{x}^{1}$ is the same as the one for $\mathbf{l}$; it suffices to consider $\mathbf{l}$.

The decisive problem is to understand the dimensions of the components of vector $\mathbf{l}$. They are to be related to our activity vector shown above, which presupposes that the period of the production of the system is one year. The annual production of corn is produced by means of $1500 \mathrm{~h}$, that of pigs by means of $250 \mathrm{~h}$, and that of oil by means of $300 \mathrm{~h}$. This leads to an extensive representation of the labour vector $\mathbf{l}^{*}$, and a reduced representation $\mathbf{I}^{* *}$ :

$$
\mathbf{I}^{*}=\left(\begin{array}{c}
\frac{1500 \mathrm{~h} / \text { year }}{150 \mathrm{~kg} \mathrm{corn} / \text { year }} \\
\frac{300 \mathrm{~h} / \text { year }}{50 \mathrm{pigs} / \text { year }} \\
\frac{600 \mathrm{~h} / \text { year }}{1001 \text { oil } / \text { year }}
\end{array}\right)=\left(\begin{array}{c}
10 \frac{\mathrm{h}}{\mathrm{kg} \mathrm{corn}} \\
6 \frac{\mathrm{h}}{\mathrm{pig}} \\
6 \frac{\mathrm{h}}{1 \text { oil }}
\end{array}\right)=\mathbf{I}^{* *}
$$

We, therefore, get two magnitudes as averages:

$\bar{l}^{*}=800 \frac{\mathrm{h}}{\text { sector }}$ and $\bar{l}^{* *}=7 \frac{1}{3} \frac{\mathrm{h}}{?}$.

The first number $\bar{l}^{*}$ is clear. The structure of production is given and $100 \mathrm{~h}$ of labour are used on average in each sector. But once we have abstracted from the concrete production in the system, the average $\bar{l}^{* *}$ becomes meaningless. For to what does the average refer? In the case of $\mathbf{I}^{*}$, we may say that the economy employs $1500+300+600 \mathrm{~h}$ of labour and distributes it to three sectors. This average and its use in our theory are invariant to changes in the unit of measurement. If, instead of reckoning in terms of kilograms, I reckon in terms of gram, I get (because the amount of corn is the same)

$$
\frac{1500 \mathrm{~h} / \text { year }}{150.000 \text { g corn } / \text { year }}
$$

The average of labour per sector remains, therefore, the same, irrespective of how I measure the commodities produced.

I may also change the unit of measurement of labour. If instead of allocating 2400 $\mathrm{h}$ of labour in total, I express the same in terms of minutes, I get 144.000 minutes per 
year. This will not affect the correlation coefficient, for this is defined according to the formula $\left(\sigma_{X}^{2}=\right.$ variance of $\left.\mathrm{X}\right)$

$$
\operatorname{cor}(X, Y)=\frac{\operatorname{cov}(X, Y)}{\sigma_{X} \sigma_{Y}} .
$$

The correlation coefficient is normalized so that the factor 60 for the transition from hours to minutes cancels. So the correlation is invariant to change in the measurement unit, here of time, while it is true that there is no such invariance in the case of the covariance. We assume the correlation to be small and then put it equal to zero as an approximation, and this will be the case, if and only if the covariance is zero. The first assumption is independent of the unit of measurement.

It is now clear why Morioka $(2019$, p. 311$)$ gets to a different result: he uses $\bar{l}^{* *}$, where the average does indeed have no meaning. The formulation used in $\mathbf{I}^{* *}$ is part of linear activity analysis and useful there, whenever activity levels are being changed, and one is looking for properties of the system, which are invariant to changes of the activity levels. But then one cannot meaningfully speak about the average labour expenditure per sector since that is precisely what is being changed. Any discussion of my covariance assumption must, of course, use the assumptions under which the postulated averages are meaningful.

We must add that the formation of average is here does not only presuppose that we keep the structure of the production of quantities fixed (for each period-the structure changes slowly with progress and effective demand from one long period to the other), but we also start from the assumption that labour is homogenous: this is one of the problems which are at the centre of attention for Marx. To regard the quantities as given is what we learned from Sraffa. There is also a more specific Marxian element in the consideration. Why is it possible at all to add the labours performed in corn production, the raising of pigs and the pumping of oil? The Marxian answer is that abstract labour is at the origin of this equalization, on the basis of which he forms the concept of simple average labour. He also distinguishes between simple and complex labour. There is no room here to discuss these sophisticated distinctions. But it may be useful to indicate the alternative. I have spoken of "strong homogeneity" to characterize the concept used by Marx and of "weak homogeneity" to distinguish it from Ricardo's notion, where labour is made homogeneous using relative wage rates as weights to add up different kinds of labour (see Essay III.4a in Schefold 1989).

Now we turn to the quantities produced and to activity balance $\mathbf{m}=\mathbf{y}-\mathbf{q}_{1}$, where $\mathbf{q}_{1}$ is the Sraffa vector. It is proportional to Sraffa's standard commodity and such that $\mathbf{y}=\mathbf{q}_{1}+\mathbf{q}_{2}+\ldots+\mathbf{q}_{n}$; the activity level vector is represented as a linear combination of the left-hand eigenvectors of $\mathbf{A}$, and the eigenvectors are normalized so that the direct sum gives $\mathbf{y}$.

To compare across industries, we introduce the gold prices pertaining to the equilibrium rate of profit $\bar{r}$. Gold is measured in grams $g$ and the price vector expressed in terms of gold is $\mathbf{p}^{*}(\bar{r})$ at the equilibrium rate of profit.

The question of the homogeneity of gold is decidedly less difficult than that of the homogeneity of labour. Marx abstracts from the cost of minting and seigniorage, 
although these loom large in the writings of other monetary economists. Our vector of activity levels above now becomes, if the prices of corn, pigs and oil are $2 \mathrm{~g}$ gold/kgcorn, $10 \mathrm{~g}$ gold/pig, $1 \mathrm{~g}$ gold/1 oil:

(corn: 300g gold, pigs: 500g gold, oil: $100 \mathrm{~g}$ gold).

The value of gross sectorial production per year is $300 \mathrm{~g}$ gold. It is clear as in the case of labour that the transition to other measurement units of the commodities does not change this average, because a change in the units of quantities will lead to a compensating change of gold prices, and the correlation is invariant to changes in the measurement of gold. The assumption that there is no correlation between the standard balance $\mathbf{m}$ and the labour balance $\mathbf{v}$ is, therefore, independent of the units chosen because it is to be related to a given structure of production, for which quantities are given.

After the discussion of the measurement units, Morioka returns to the discussion of the assumptions themselves. I am well aware of the difficulties of the principle of indifference and the Bertrand paradox, but I had said simply: There is no reason why $\mathbf{m}$ and $\mathbf{v}$ should be correlated. One might reply: The correlation between $\mathbf{m}$ and $\mathbf{v}$ is measurable. Why is not each possible value of the correlation a priori equally likely? I have replied above: If there is a spurious correlation, it should be explained. This does not mean, of course, that every spurious correlation in reality could be explained; it may simply be due to hazard. But when we are discussing economic models, we should advance theoretical reasons why we postulate a correlation or the lack thereof. For economists as theorists, there must be reasons. And as long as no reason is advanced for a positive or negative correlation, it is an at least licit, and, by the principle of indifference, even plausible assumption that there is no such correlation.

As in every case of abstract reasoning, the stylized character of the assumptions excludes some known possibilities. For instance, Morioka mentions that the surplus vector must be positive by virtue of our assumptions, while Sraffa took care to distinguish between basics and non-basics. True, there is room for zero entries in the input matrix, but no room for non-basics in our present theory of random matrices. The theory might perhaps be extended to take account of them, but that is not how I would like to defend myself in this case. Indeed, in Marx, if the system is stationary, constant capital will not be consumed and will not be part of the surplus vector $\mathbf{s}$. The logic of the analysis of random matrices has compelled us to abandon the distinction of basics and non-basics and the corresponding distinction in Marx to concentrate on another aspect, but this is no more an argument against the model than the existence of monopolies in theory and practice is an argument not to analyse the perfect competition.

Morioka tries yet another attack. The covariance argument is only used to prove $\mathbf{m v}=0$ (we may say $\mathbf{y v}=\mathbf{m v}=0$ ) and $\mathbf{s v}=0$ : "There seems to be no reason that both the gross and surplus production vectors must be orthogonal to vector $\mathbf{v}$, which is determined only by technological conditions" (Morioka 2019, p. 312).

I could reply that the assumptions can indeed not so easily be formulated in terms of the inner products of the vectors directly and that it is more meaningful to found 
them on the correlations. Nonetheless, we can try to show that, even on this basis, we remain within the realm of assumptions that are, certainly, "special", but not unusual in classical theory. It is elementary that the inner products can be written as $\mathbf{y v}=|\mathbf{y}||\mathbf{v}| \cos \varphi$ and $\mathbf{s v}=|\mathbf{s}||\mathbf{v}| \cos \psi$, where $\varphi$ is the angle between vectors $\mathbf{y}$ and $\mathbf{v}$ and $\psi$ the angle between vectors $\mathbf{S}$ and $\mathbf{v}$. We have defined $\mathbf{v}$ as the labour balance, which must have positive and negative entries since the average of the components of this vector is equal to zero, as we have seen, if the one-industry system is of even capital composition. Since $\mathbf{y}$ and $\mathbf{s}$ are positive, the orthogonality is, therefore, possible. If we look at the formula for the inner product, we may argue that the absolute value of $\mathbf{v}$ is small, using Ricardo's argument that the deviation of prices from values is not large (remember his famous estimate that the difference amounts only to $7 \%$, which caused other people to speak of the "93\% labour theory of value"). This would be the traditional reason for a small inner product. To argue that the angles $\varphi$ and $\psi$ are close to orthogonal then means to return to the correlation assumption in a new form. One would thus get a product of two small quantities, which could be considered as very small. It is quite true that the assumption that the correlations are equal to zero is neither logically necessary nor empirically confirmed, but it has some plausibility and is a mean to rationalize, why Marx insisted on $P=M$ and the neoclassical authors on a given quantity of capital in nominal terms prior to distribution.

\section{Mori: a Ricardian answer to a Marxian question}

I already have replied to Kenji Mori's stimulating comments in the printed version of my paper (see Schefold 2019, pp. 277, 291, 292). I repeat briefly in the light of the previous discussion. I agree with Mori that Marx does not equalize the total gross products in terms of prices and values simply by choosing a numéraire, but that his measure for prices and values is provided by money, which means gold (we need not discuss here his transition from gold as the general equivalent to coined gold as money and the substitutes thereof). Gross production in both evaluations, values and prices of production, is equal to an imaginary amount of gold, the purchasing power of which should not be affected by the transformation. The simple solution here seems to me to be the same as in Ricardo, when he discusses what could represent his ideal standard of value. It is produced by an industry of average capital composition, that is, expressing it in modern terms, an average of the capital intensities of all industries. He thought that gold production might represent such an average satisfactorily, and the Marxian assertion about gold in the transformation process really implies that he made — consciously or not — the same assumption. The capital composition of gold production must be equal to the capital composition of total production, that is, of $\mathbf{y}$. This is not possible for one industry taken in isolation; if the argument is to be reproduced rigorously, one has to take recourse to a composite industry. Let $\gamma \mathbf{y}$ be the activity level vector of the gold industry, with capital goods $\gamma \mathbf{y A}$ and with labour $\gamma \mathbf{y l}$. Value and price of the gross product of this industry are then $\gamma \mathbf{y} \overline{\mathbf{p}}(r)=\gamma$. The idea is general and independent of random systems. The analytical reconstruction of the Marxian argument is here not difficult. 
Kenji Mori also observes that Marx "regarded the "principle of multiples"... as one of the most characteristic features of the "machinery system" " (Mori 2019, p. 316). The production of machines requires the combination of individual parts and, prior to that, of raw materials, in determinate quantities in each industry. This is what input-output systems show in the small, both theoretically and empirically. But this does not mean that one cannot take a different view with regard to large ensembles as in physics or astronomy. The movement of a few particles is quite determinate, while the movements in a gas or in a galaxy can only be described by statistical mechanics. The number of sectors in real economies is not as large as the number of particles of a gas in a container in a laboratory, but why should one not try to grasp economic reality by sometimes looking at it in the small and, for other questions, in the large? I expect that economists will get used to assumptions such as the ones used here in the same way as they once became used to adopting consumption functions or similar constructs, which had first appeared strange. I think one should give credit to Marx, whose originality was such that we need quite modern concepts for the analytical reconstruction of his ideas, alongside others that refer to older economists like Ricardo.

In conclusion, I should like to thank my commentators once more for their challenging observations and suggestions. It is true that special assumptions were needed for this reconstruction of the Marxian transformation of values into prices. It at least provides some justification for the Marxian approach, which is neither an absolute truth nor quite wrong. He remains one of the great authors of our subject, and we can integrate his contribution in the spirit of modern pluralism.

Acknowledgments Open Access funding provided by Projekt DEAL.

\section{Compliance with ethical standards}

Conflict of interest There is no conflict of interest regarding this paper (160420 BS/ba).

Open Access This article is licensed under a Creative Commons Attribution 4.0 International License, which permits use, sharing, adaptation, distribution and reproduction in any medium or format, as long as you give appropriate credit to the original author(s) and the source, provide a link to the Creative Commons licence, and indicate if changes were made. The images or other third party material in this article are included in the article's Creative Commons licence, unless indicated otherwise in a credit line to the material. If material is not included in the article's Creative Commons licence and your intended use is not permitted by statutory regulation or exceeds the permitted use, you will need to obtain permission directly from the copyright holder. To view a copy of this licence, visit http://creativecommons.org/licen ses/by/4.0/.

\section{References}

Mori K (2019) A comparison of the stochastic approach to the transformation problem with Marx's original assumptions. Evolut Inst Econ Rev 16(2):315-318 (Japan Association for Evolutionary Economics)

Morioka M (2019) The transformation problem under positive rank one input matrices: on a new approach by Schefold. Evolut Inst Econ Rev 16(2):303-313 (Japan Association for Evolutionary Economics) 
Schefold B (1989) Mr. Sraffa on joint production and other essays. Unwin \& Hyman, Routledge

Schefold B (2013) Approximate surrogate production functions. Camb J Econ 37(5):947-983

Schefold B (2016a) Profits equal surplus value on average and the significance of this result for the Marxian theory of accumulation: being a new contribution to Engels' prize essay competition, based on random matrices and on manuscripts recently published in the MEGA for the first time. Camb J Econ 40(1):165-199

Schefold, B (2016) Marx, the production function and the old neoclassical equilibrium. Workable under the same assumptions? With an appendix on the likelihood of reswitching and of Wicksell effects. Centro Sraffa Working Papers, n.18, March 2016, 57 pp. Online as Centro Sraffa working papers

Schefold B (2019) The transformation of values into prices on the basis of random systems revisited. Evolut Inst Econ Rev 16(2):261-302 (Japan Association for Evolutionary Economics)

Yagi K (2019) Special feature: workshop on random system solution of the transformation problem: discussion with Prof Bertram Schefold. Evolut Inst Econ Rev 16(2):259-260 (Japan Association for Evolutionary Economics)

Yamazaki Y (2019) Profit and value in a random system: interpretation of Professor Schefold's 2016 article. Evolut Inst Econ Rev 16(2):319-325 (Japan Association for Evolutionary Economics)

Publisher's Note Springer Nature remains neutral with regard to jurisdictional claims in published maps and institutional affiliations. 